\title{
Immunofluorescence Staining of WT-1/Podocalyxin on Mouse Kidney Sections
}

Carole Henique $^{1, *}$, Olivia Lenoir ${ }^{2}$ and Pierre-Louis Tharaux ${ }^{2,3, *}$

${ }^{1}$ Institut Mondor de Recherche Biomédicale (IMRB), Inserm U955, Université Paris Est Créteil UPEC, Equipe 21, Créteil, France; ${ }^{2}$ Paris Cardiovascular Centre (PARCC), Inserm U970, Université Paris Descartes, Sorbonne Paris Cité, Paris, France; ${ }^{3}$ Renal Division, Georges Pompidou European Hospital, Assistance Publique-Hôpitaux de Paris and Paris Descartes University, Paris, France *For correspondence: carole.henique@inserm.fr; pierre-louis.tharaux@inserm.fr

[Abstract] In glomerular diseases, podocytes are one type of cells involved in dysfunction of glomerular filtration. In these conditions, podocyte proteins expression change. Therefore, immunofluorescent staining of podocytes can be performed to visualize podocyte localization of proteins of interest. In this protocol, we detail a method to stain podocytes with a specific marker WT-1 (Wilms Tumor-1) for "in situ" podocyte analysis by immunofluorescent microscopy, more informative technique than other techniques (as Western blot).

Keywords: Immunofluorescence, Staining, Podocyte, Mouse, IF, WT-1, Podocalyxin, Kidney

[Background] In vivo, mature podocytes are glomerular epithelial cells that are normally terminally differentiated (Pavenstadt et al., 2003). Under pathological conditions, podocytes may undergo mitosis and proliferate. For example, crescentic glomerulonephritis is an exceptional condition where podocytes undergo a dysregulation of their differentiated phenotype and start to proliferate and migrate, resulting in the so-called extracapillary glomerulopathy. Several studies have shown involvement of podocytes in crescentic glomerulonephritis (Moeller et al., 2004).

Wilm's tumor 1 (WT-1) is a zinc finger transcription factor present in nucleus of podocyte. Podocalyxin is a sialoglycoprotein constituting the glycocalyx of podocytes and endothelial cells in glomerulus. The expression of WT1 and podocalyxin are not decreased in early-stage of glomerular damage compared to proteins of slit diaphragm as podocin or nephrin. Moreover, WT1 is useful to evaluating podocyte number and density under different circumstances. The advantages to use podocalyxin staining are i) to analyze the glomerular morphology, ii) to quantify flocculus area to perform WT1+ cells / flocculus area ratio; iii) to ensure that WT1+ cells are podocytes and no other cells as parietal epithelial cells which could express WT1 in pathophysiological context.

Our protocol can be used in crescentic glomerulonephritis (Henique et al., 2017) but also in studies on different glomerulopathies as hypertensive nephropathy.

\section{Materials and Reagents}

1. Slides Menzel-Gläser Superfrost ${ }^{\circledR}$ PLUS $25 \times 75 \times 1.0$ mm (Thermo Fisher Scientific, catalog number: J1800AMNZ) 
2. Cover slip (Knittel Glass, 24 x $50 \mathrm{~mm}$, catalog number: 023/40)

3. PAP pen (DakoPen, Dako, catalog number: S2002) or equivalent

4. Tissue Tek container/Staining Dish green (solvent-resistant) (Sakura, catalog number: SKU4456)

5. Paper towels

6. Aluminum foil

7. $0.2 \mu \mathrm{m}$ syringe filter (VWR, catalog number: 514-0060)

8. Primary antibody

a. Rabbit anti-Wilms Tumor Protein (WT-1) (dilution ratio 1:100, put $1 \mu$ l of antibody with $99 \mu \mathrm{l}$ of blocking solution) (clone [CAN-R9(IHC)-56-2]) (Abcam, catalog number: ab89901)

b. Biotinylated goat anti-mouse podocalyxin (dilution ratio 1:500, put $1 \mu \mathrm{l}$ of antibody with 499 $\mu \mathrm{l}$ of blocking solution) (R\&D, catalog number: BAF1556)

9. Secondary antibody

a. Alexa Fluor 594 donkey anti-rabbit (dilution ratio 1:400, put $1 \mu \mathrm{l}$ of antibody with $399 \mu \mathrm{l}$ of blocking solution) (Thermo Fisher Scientific, catalog number: A21207)

b. Alexa Fluor 488 donkey-anti-goat (dilution ratio 1:400, put $1 \mu \mathrm{l}$ of antibody with $399 \mu \mathrm{l}$ of blocking solution) (Thermo Fisher Scientific, catalog number: A11055)

c. Streptavidin, Alexa Fluor 488 (Thermo Fisher Scientific, catalog number: S32354)

10. Xylene (Carlo Erba, catalog number: 528251)

11. Ethanol absolute (Carlo Erba, catalog number: 3086072)

12. Target retrieval solution (Dako, catalog number: S1699)

13. Fluorescence Mounting Medium (Dako, catalog number: S3023)

14. DAPI solution (1 mg/ml) (Thermo Fisher Scientific, catalog number: 62248)

15. Clear Nail Polish (available at local drug store)

16. Phosphate Buffer Saline 10x (Euromedex, catalog number: ET330)

17. Triton ${ }^{\mathrm{TM}} \mathrm{X}-100$ (Sigma-Aldrich, catalog number: $\mathrm{X} 100$ )

18. Tween ${ }^{\circledR} 20$ (Sigma-Aldrich, catalog number: P2287)

19. Bovine serum albumin (BSA) (Sigma-Aldrich, catalog number: A2058)

20. Sudan Black $B$ (Acros organics, catalog number: 190160250)

21. Tris (Euromedex, catalog number: 200923-A)

22. $\mathrm{NaCl}$ (Euromedex, catalog number: 1112-A)

23. Tris-buffered saline (TBS) (see Recipes)

24. TBS-Tween (1x) (see Recipes)

25. Permeabilization solution (see Recipes)

26. Blocking solution (see Recipes)

27. Sundan Black B solution (see Recipes) 


\section{Equipment}

1. Axioimager Z1 apoptome (Zeiss)

2. Pressure cooker, any pressure cooker can be used, here Decloaking chamber (Biocare medical, model: DC2008)

3. Benchtop orbital shaker (Stuart scientific gyro rocker, model: STR9)

4. $4{ }^{\circ} \mathrm{C}$ refrigerator

\section{Software}

1. Axiovision microscopy software (Version AxioVs40 V 4.8.2.0, Zeiss)

\section{Procedure}

1. Prepare Xylene (2 containers), Ethanol (100\%, 95\%, 70\%) and distilled water baths with $300 \mathrm{ml}$ of solution in staining dish.

Note: Staining works well in freshly cut tissue.

2. Deparaffinate Formalin Fixed Paraffin Embedded (FFPE) mouse kidney. Immerse sections in:

$\begin{array}{ll}\text { Xylene } & 5 \mathrm{~min} \\ \text { Xylene } & 5 \mathrm{~min}\end{array}$

Note: Before moving to ethanol step, make sure to completely deparaffinize the sections. If there is still traces of wax immerse in Xylene for an extra $5 \mathrm{~min}$.

$\begin{array}{ll}\text { Ethanol } 100 \% & 3 \mathrm{~min} \\ \text { Ethanol } 95 \% & 3 \mathrm{~min} \\ \text { Ethanol } 70 \% & 3 \mathrm{~min} \\ \text { Distilled water } & \end{array}$

3. Prepare $300 \mathrm{ml}$ of freshly antigen retrieval solution according to the manufacturer instructions.

4. Turn on the power button of pressure cooker. Set the temperature and time by pressing the "Display Set" button until the SP1 LED light comes on. Use the up or down arrows to adjust the temperature to the desired retrieval temperature $\left(95^{\circ} \mathrm{C}\right)$. Press the "Display Set" button once and the SP1 time will display. Use the up and down arrows to adjust to the proper time.

5. Immerse the slides in antigen retrieval solution in Tissue Tek racks in staining container (about $300 \mathrm{ml}$ ). Arrange Tissue Tek Containers on top of the heat shield off-center to avoid heat concentration. Containers must not be covered. The decloaking chamber can contain up to 4 racks, each containing 24 slides.

Note: Do not use glass containers.

a. Align the shell and pan handles.

b. Match the lid etching "OPEN" with the white dot on the pan handle. 
c. Hold both the shell and pan handle with your left hand while turning the lid clockwise with the right hand until the lid etching that reads "CLOSED" is aligned with the white dot of the pan handle on the left side.

d. The metal tabs on the lid should be tightly seated against the pan's lip.

e. Turn the instrument on by flipping the red toggle to the right of the control panel to the "ON" position.

f. Press "DISPLAY SET" once, until the SP1 LED illuminates.

g. The user can scroll through the instrument settings by pushing the "DISPLAY SET" button multiple times.

h. Push "Start/Stop" button to initiate the programmed run.

i. When the program is finished, visually confirm that the pressure has dropped to 0 (zero) psi on the pressure gauge.

j. Toggle the weight (petcock) to release any residual pressure. Turn the unit off.

k. Open the lid with the steam directed away from yourself.

I. Leave the containers in the Decloaking Chamber to cool down for $10 \mathrm{~min}$.

6. Carefully remove the Tissue Tek container from the pressure cooker and place it at room temperature (RT) for 20 min to cool down. The final temperature should be room temperature (RT).

7. Remove the slides out of Tissue Tek container and circumscribe the kidney slice on the glass slide with a PAP pen (liquid blocker).

Note: Do not let the tissue dry.

8. Prepare permeabilization solution, add it on slides by gently droping it within the circumscribed area and make sure the tissue is fully covered and incubate the slides for $15 \mathrm{~min}$ at RT.

9. Wash slides 3 times for 5 min each in $1 \times$ TBS-Tween in a slide rack in green staining dish (with about $300 \mathrm{ml}$ of buffer), make sure the tissue is immersed in buffer. Place slides container on a benchtop orbital shaker (75 to $100 \mathrm{rpm}$ ) during each wash.

10. Prepare blocking solution, add on slides and incubate the slides for $60 \mathrm{~min}$ at RT.

11. Prepare the anti-WT1 and anti-podocalyxin primary antibody in blocking solution (diluted with blocking buffer at 1:100 for WT-1, 1:500 for podocalyxin).

12. Throw away blocking solution by dumping the blocking solution from the slides onto paper towels and incubate the slides directly with primary antibody overnight in a humidified chamber at $4{ }^{\circ} \mathrm{C}$.

Note: For assembly of a humidified chamber, you need: a plastic box with lid, plastic pipettes, paper towel and distilled water. Moisten the paper thoroughly with distilled water and put into the box. Put two plastic pipettes on the paper to create a support for the slides. Put antibody solution on slides and put the lid on the box and incubate for the desired time.

13. After overnight incubation, wash slides 3 times for 5 min each in $1 \times$ TBS-Tween in green staining dish (with about $300 \mathrm{ml}$ of buffer).

14. Prepare secondary antibody (about $50 \mu \mathrm{l}$ for one section, diluted with blocking solution at 1:400) 
and add it directly on slides. Incubate slides at RT for 60 min in a humidified chamber wrapped in aluminum foil to being sure to protect from light.

Note: The anti-podocalyxin is a biotinylated antibody thus it is possible to use a fluorescent streptavidin as secondary antibody (Streptavidin, Alexa Fluor 488).

15. To visualize DNA/nuclei, prepare DAPI solution $(1 \mu \mathrm{g} / \mathrm{ml}$ ) (about $50 \mu \mathrm{l}$ for one section) in $1 \mathrm{x}$ PBS.

16. Throw away secondary antibody solution and apply DAPI solution directly on slides for 5 min at RT.

17. Then, wash slides 3 times each for 10 min in $1 x$ TBS-Tween in green staining dish (with about $300 \mathrm{ml}$ of buffer) at RT.

18. Incubate with filtered Sudan Black B solution directly on slides for $30 \mathrm{~s}$ in the dark at RT.

19. Wash slides in green staining dish for 10 min under flowing tap water.

20. Add a small amount of fluorescent mounting medium (1 drop) and place a cover slip over the specimen, avoiding bubbles.

21. Use clear nail polish to seal the sides of the cover glass to the slide.

22. Let slides incubate at $4{ }^{\circ} \mathrm{C}$ overnight in the dark.

23. After imaging, slide can be stored at $4{ }^{\circ} \mathrm{C}$ in the dark for at least 6 months with minimal loss of fluorescence.

\section{Data analysis}

For an example of an immunofluorescent picture obtained with this protocol, see Figures 1 and 2.

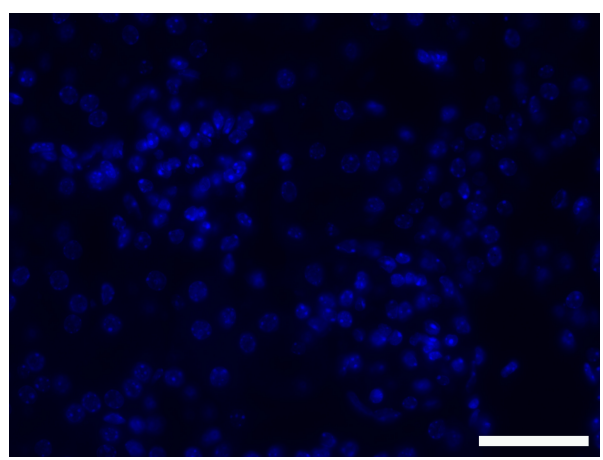

Figure 1. Negative control with secondary antibody only. The picture shows that the secondary antibodies do not cause any background. Scale bar: $50 \mu \mathrm{m}$. 


\section{biö-protocol
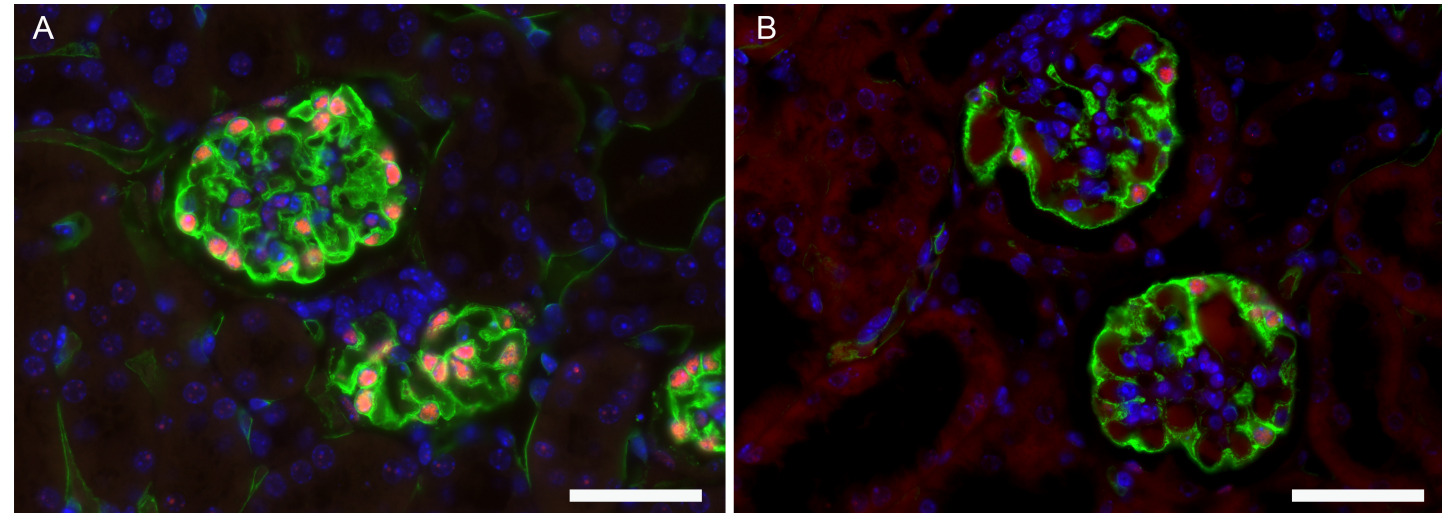

Figure 2. Immunofluorescent image of glomerulus with visualization of podocytes with

markers. WT-1 (red) and podocalyxin (green). Nuclei are stained with DAPI (blue). A. Normal kidney from male mouse (C57BI/6J, 12 weeks). B. Kidney from mouse with hypertension (male, C57BI/6J). These pictures show a decrease in WT1 and podocalyxin staining suggesting a loss of podocytes and dilated capillaries. Scale bars: $50 \mu \mathrm{m}$.

\section{$\underline{\text { Recipes }}$}

1. Tris-buffered saline (TBS)

$25 \mathrm{mM}$ Tris

$0.15 \mathrm{M} \mathrm{NaCl}$

Final $\mathrm{pH}$ of solution should be 7.2 to 7.5

2. TBS-Tween (1x)

TBS containing $0.1 \%$ Tween $^{\circledR} 20$

3. Permeabilization buffer

TBS containing $0.1 \%$ Triton $^{\mathrm{TM}} \mathrm{X}-100$

4. Blocking solution

TBS-Tween $1 x$ containing 5\% BSA

5. Sudan Black $B$ solution

0.1\% Sudan Black B

$70 \%$ ethanol

Note: Sudan Black B solution was made up in the dark and need to be filtered ( $0.2 \mu \mathrm{m}$ syringe filter) to remove undissolved dye.

\section{Acknowledgments}

We thank members of Tharaux lab, as well as histology core facility at PARCC for helpful input. This work was supported by Inserm, the European Research Council-ERC Grant 107037 to P-L.T. and the Association des maladies d'un syndrome Néphrotique. We are grateful to the French National Agency for Research (ANR grant "SWITCHES" to P-L.T.) for supporting C. Henique. 


\section{Competing interests}

The authors declare no competing financial interests.

\section{$\underline{\text { References }}$}

1. Henique, C., Bollee, G., Loyer, X., Grahammer, F., Dhaun, N., Camus, M., Vernerey, J., Guyonnet, L., Gaillard, F., Lazareth, H., Meyer, C., Bensaada, I., Legres, L., Satoh, T., Akira, S., Bruneval, P., Dimmeler, S., Tedgui, A., Karras, A., Thervet, E., Nochy, D., Huber, T. B., Mesnard, L., Lenoir, O. and Tharaux, P. L. (2017). Genetic and pharmacological inhibition of microRNA-92a maintains podocyte cell cycle quiescence and limits crescentic glomerulonephritis. Nat Commun 8(1): 1829.

2. Moeller, M. J., Soofi, A., Hartmann, I., Le Hir, M., Wiggins, R., Kriz, W. and Holzman, L. B. (2004). Podocytes populate cellular crescents in a murine model of inflammatory glomerulonephritis. J Am Soc Nephrol 15(1): 61-67.

3. Pavenstadt, H., Kriz, W. and Kretzler, M. (2003). Cell biology of the glomerular podocyte. Physiol Rev 83(1): 253-307. 